\title{
A NOTE ON CONVEXITY IN EUCLIDEAN $n$-SPACE
}

\author{
HERMAN RUBIN AND OSCAR WESLER
}

If a subset $S$ of a real linear space is convex (with every two points $x_{1}, x_{2}$ of $S$, the entire line segment $\lambda x_{1}+(1-\lambda) x_{2}, 0 \leqq \lambda \leqq 1$, is contained in $S$ ) then it is also closed under the operation of taking finite convex linear combinations. For any set $R$ in the space, the set of all finite convex linear combinations is the convex hull of $R$ (the smallest convex set containing $R$ ). If the space has finite dimension $n$, it is well known that it is enough to mix at most $n+1$ points at a time to get the convex hull. If the space is topological, convergent mixtures of countably many points of $R$, as well as mean values of arbitrary measures on $R$, may also be taken. The question of how much more than the convex hull one gets thereby arises. It is well known that in an arbitrary locally convex topological space one stays in the closure of the convex hull. We show in this paper that in Euclidean $n$-space the interesting and useful fact is that one never gets out of the convex hull itself. A counter-example in Hilbert Space shows that infinite mixtures may take one outside the convex hull when the space is not finite dimensional. It will suffice to state our theorem in the following form.

Theorem. Let $S$ be a convex set in Euclidean $n$-space, $E_{n}$, and let $y \in E_{n}$ be a point representable as $y=\sum_{i=1}^{\infty} \lambda_{i} x_{i}$ where each $\lambda_{i} \geqq 0$, each $x_{i} \in S$, and $\sum_{i=1}^{\infty} \lambda_{i}=1 ;$ or, more generally, representable as $y=\int_{S} x d \mu(x)$ where $\mu$ is an arbitrary probability measure on $S$. Then $y$ belongs to $S$.

Proof. Note that the convergence of the series is not required to be absolute and can even be an arbitrary Banach limit. The same for the integrals; we only require, as a necessary condition for the integrals to make sense, that whatever the probability measure $\mu$, the measurable subsets of $S$ are taken to contain the intersections of $S$ with the Borel sets in $E_{n}$ as a sub- $\sigma$-algebra. A simple proof for countable combinations was written down by us for inclusion as Theorem 2.4.1 in [1]. A proof for general measures can be constructed without too much difficulty along almost identical lines. We present here an even simpler proof.

Let $L$ be a hyperplane of lowest dimension $p$ such that $\mu(L \cap S)=1$. Clearly, we need confine our attention only to the convex subset $L \cap S$ of $S$. If $p=0$, there is nothing to prove. Suppose therefore that

Received by the editors December 17, 1957. 
$p$ is positive, and that $y$ does not belong to $S$. Then $y$ is a relative boundary point of $L \cap S$, so that there exists a supporting hyperplane $L_{1}$ of dimension $p-1$ in $L$ at the point $y$. We assert that $\mu\left(L_{1} \cap S\right)=1$, for otherwise the mean value $y$ would clearly lie to one side of $L_{1}$ whereas in fact $y$ belongs to $L_{1}$. But this contradicts the minimal dimensionality of $L$, hence $y$ must belong to $S$ (indeed $y$ is a relative inner point of $L \cap S$ ) and the proof is complete.

To see that the result may break down for infinite dimensions, consider in Hilbert Space (a point here is a sequence of real numbers) the set $S$ of all points all but finitely many of whose coordinates are equal to zero. $S$ is clearly convex. However, the convergent mixture $x=\sum_{i=1}^{\infty} x_{i} / 2^{i}$, where $x_{i}=\left\{\delta_{n i}\right\}$, is the point $\left\{1 / 2^{n}\right\}$ which does not belong to $S$.

\section{BIBLIOGRAPHY}

1. D. Blackwell and M. A. Girshick, Theory of games and statistical decisions, John Wiley and Sons, New York, 1954, pp. 48-49.

UNIVERSITY OF OREGON AND

UNIVERSITY OF MiCHIGAN 